\title{
Fabrication and Application of Low Cost Flexible Film-Based Sensors to Environmental and Biomedical Monitoring Scenarios
}

\author{
Vladimir Laukhin ${ }^{1,2,3}$, Victor Lebedev ${ }^{1}$, Elena Laukhina ${ }^{1,2(\bowtie)}$, \\ Andrey Somov ${ }^{4}$, Alexander Baranov ${ }^{5}$, Concepcio Rovira ${ }^{1,2}$, \\ and Jaume Veciana ${ }^{1,2}$ \\ ${ }^{1}$ Institut de Ciencia de Materials de Barcelona (ICMAB-CSIC), Campus UAB, \\ Bellaterra 08193, Spain \\ laukhina@icmab.es \\ ${ }^{2}$ CIBER de Bioingeniería, Biomateriales y Nanomedicina (CIBER-BBN), \\ Madrid, Spain \\ ${ }^{3}$ Institució Catalana de Recerca i Estudis Avançats (ICREA), Barcelona, Spain \\ ${ }^{4}$ CREATE-NET, Trento, Italy \\ 5 'MATI'-Russian State Technological University, Moscow, Russia
}

\begin{abstract}
The paper describes the development of flexible lightweight highly sensitive film-based sensors capable of monitoring pressure, deformation, temperature and humidity. In particular, we present a family of the developed simple devices that successfully adopted polycarbonate films covered with organic molecular conductors as conductive sensing components. Proof-ofconcept experiments with these prototypes demonstrate that such bi layer films are promising as sensing devices for the environment and biomedical monitoring. Besides, we present the interfacing of the flexible film-based sensor with a wireless sensor node and evaluate the sensing capability of this system in a real monitoring scenario.
\end{abstract}

Keywords: Flexible sensors · Organic molecular conductors $\cdot$ Wireless Sensor Network $\cdot$ Environmental monitoring $\cdot$ Biomedical monitoring

\section{Introduction}

The development of flexible, lightweight, conducting materials, whose electrical transport properties strongly respond to delicate strain, brings great opportunities in the field of strain, pressure, bending and temperature sensors for their applications in smart textiles, robotic interfaces and body sensing devices [1-3]. Here it should be noted that the modern working up wireless monitoring technologies take an active interest in using such conductive sensing materials. The wireless monitoring sensing systems based on the Wireless Sensor Network paradigm (WSN) enables a user to perform sensing measurements of a physical phenomena, process the data and send them to a user or machine over the network. WSNs have been already used in a large number of monitoring applications, including wildlife monitoring [4] and fire detection [5, 6], noise sensing in urban areas [7] and health monitoring [8]. 
Until now the WSN nodes typically employ the off-the-shelf sensors. However, organic film sensors introduce a number of advantages over the off-the-shelf sensing devices [2]. Covering polymeric films with conventional metals and semiconductors is one of the traditional approaches to engineering flexible lightweight sensors [9]. Thus, Huang et al. [10] described a flexible thermistor fabricated by printing a square $\mathrm{NiO}$ thin film on a polyimide film. Such flexible Bi Layer (BL) sensors demonstrate a good sensitivity and fast response. Unfortunately, these flexible sensing materials demonstrate a common drawback: poor adhesion of conventional metals and their oxides to polymers. This disadvantage results in a low binding between inorganic sensing layers and plastic supports. With organic conductors one may overcome the problem. It was showed that conducting polymers can be successfully put to sensing technology [11]; the reported flexible capacitive-type humidity and temperature sensors - cellulosepolypyrrole nanocomposites - are ones of the promising examples of all-organic flexible sensors [12]. However, conducting polymers are not stable materials: their electronic properties are often unstable towards atmospheric moisture [13]. Moreover, they are expensive due to the high manufacturing costs. In this context, organic molecular conductors $(\mathrm{BEDT}-\mathrm{TTF})_{2} \mathrm{X}$, where $\mathrm{BEDT}-\mathrm{TTF}=$ bis(ethylenedithio) tetrathiafulvalen (Fig. 1) and ' $\mathrm{X}$ ' is trihalide ions [14, 15] hold a great interest for sensor engineering due to important material properties, such as lightweight, stability, conductivity and high piezoresistivity.

It should be noted that the (001) oriented conducting layer of the highly piezoresistive $\beta$-(BEDT-TTF $)_{2} \mathrm{I}_{3}$ metal can be formed on the surface of a polymeric film by a simple procedure carried out at moderated temperature [16-18]. This stimulated us to harness the BL films - polycarbonate/(BEDT-TTF) $)_{2} \mathrm{X}$ - for engineering sensing systems capable to detect ultra-small deformations or pressure. Recently, we have demonstrated that another BL film: polycarbonate/(001) oriented $\alpha$-(BEDT-TTF) $)_{2} \mathrm{I}_{3}$, can be reproducibly prepared as a temperature independent strain sensing material [19]. With the successful fabrication of the first prototype for a breathing control (Fig. 2, device 1) [18] the integration of developed conductive BL films as active components in flexible strain sensors is being evaluated. Recently, we have developed a BL thermistor: polycarbonate/ $\alpha^{\prime}-(\mathrm{BEDT}-\mathrm{TTF})_{2} \mathrm{I}_{\mathrm{x}} \mathrm{Br}_{3-\mathrm{x}}$, were $\alpha^{\prime}-(\mathrm{BEDT}-\mathrm{TTF})_{2} \mathrm{I}_{\mathrm{x}} \mathrm{Br}_{3-\mathrm{x}}$ which is organic molecular semiconductor whose electrical resistance is highly sensitive to temperature [20]. In the body temperature range its temperature coefficient of resistance (TCR) is $-1.4 \% /$ degree. This value is four times larger than that reported for the platinum thermometer $\left(\mathrm{TCRPt}_{111}=0.3\right)$ [21].

In this work we present our recent advances in the development of flexible film-based sensors [18, 20, 22], demonstrate their sensing capabilities in wearable

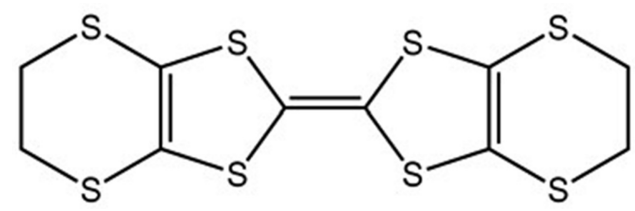

Fig. 1. Skeletal formula of bis(ethylenedithio)tetrathiafulvalen (BEDT-TTF) 


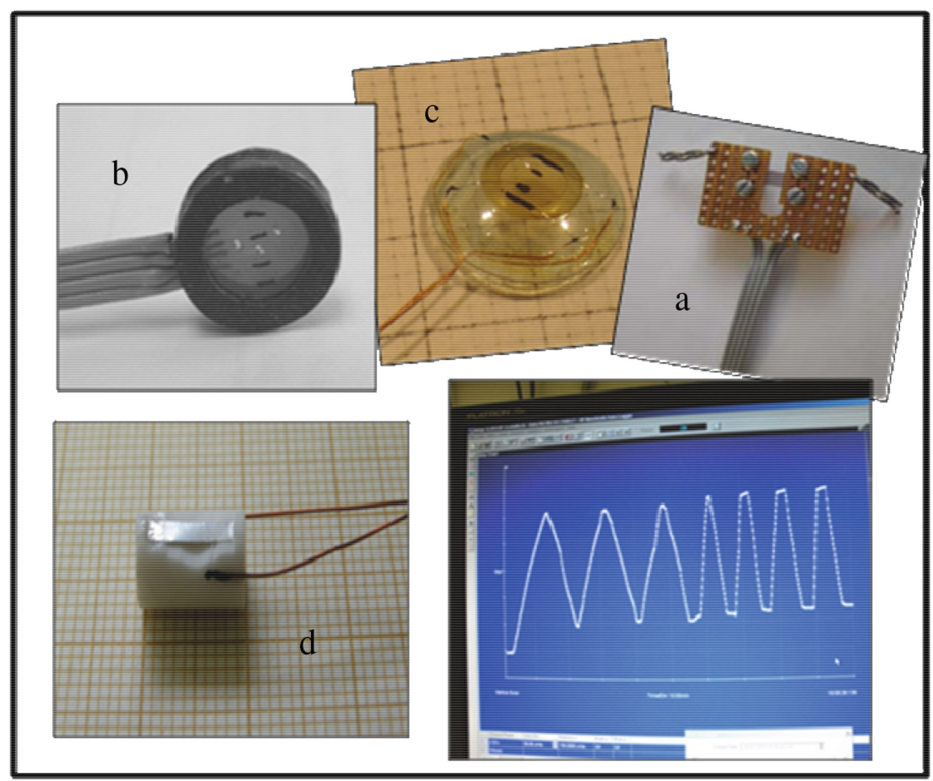

Fig. 2. Photos of some developed devices capable of monitoring the body tissue movements: (a) breathing sensor, (b) blood pulse sensor, (c) IOP sensing contact lens, and (d) pressure sensing element.

biomedical and environmental monitoring scenarios as well as their interfacing with a wireless sensing device to increase the number of potential autonomous monitoring applications.

The paper is organized as follows: in Sect. 2 we summarize the developed sensors and evaluate their performance. Next we explore the opportunity of the flexible film-based sensor interfacing with a wireless sensor node and apply this system in a real monitoring scenario in Sect. 3. Finally, we provide our conclusions and discuss our future work in Sect. 4.

\section{Development of Sensing Devices}

\subsection{Flexible Piezoresistive Sensors Controlling the Body Tissue Movement}

In this section, we discuss the development of the sensors as well as demonstrate and discuss their performance. Figure 2 presents the prototypes of sensing devices developed for the body tissue movement monitoring. In next sections we discuss each sensing device in details.

\subsubsection{Breathing Sensor}

The breathing sensor is shown in Fig. 2(a). This prototype contains the BL film: polycarbonate/ $\beta-(B E D T-T T F)_{2} I_{3}$ as a tenzo-resistive diaphragm on springy plastic 


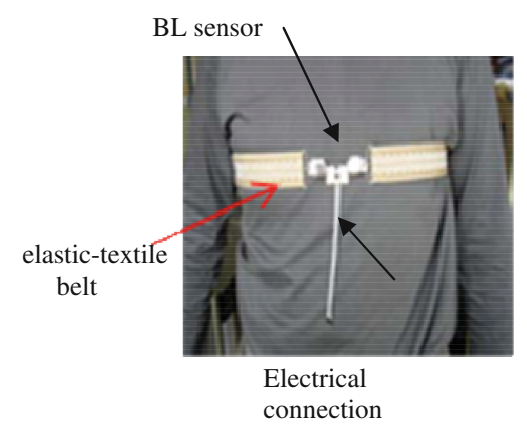

(a)

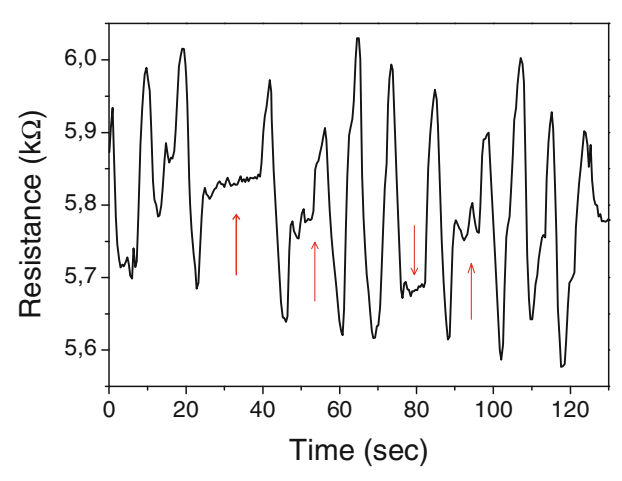

(b)

Fig. 3. (a) Photo images of the breathing sensor location on the breast of a volunteer for monitoring his breathing and (b) sensor electrical resistance response to the breathing of a volunteer (red arrows correspond to holding up his breathing) (Color figure online)

$U$-shape plate, which is attached to an elastic-textile belt (see Fig. 3a). The resistance response of the sensor to strain provoked by the breathing is measured by a four probes dc method. Data are gathered for different persons and time periods. Figure 3 a shows that the breathing movement results in the oscillated resistance curve. A relatively long time periods between the oscillations, observed in Fig. 3a (left), correspond to holding up breathing.

\subsubsection{Blood Pulse Sensor}

Blood pulse sensor is presented in Fig. 2b. The membrane fabricated from the BL film: polycarbonate/ $\beta$-(BEDT-TTF $)_{2} \mathrm{I}_{3}$, is equipped with electrical contacts and fixed between two rigid plastic rings. To measure the pulse, the device is fixed on the body of a volunteer. The resistance response of the sensor to strain provoked by pulse movement is measured by a four probes dc method. Data are gathered from different persons and time periods (Table 1).

Figure 4 shows that the pulse movement, which resulted in an oscillated resistance curve, can be easily recorded and analyzed. Additionally Fig. 4 demonstrates that the sensor electrical responses for two persons - " $V$." and " $L$." show nonsymmetrical oscillations. Moreover, in the case of " $L$." nonsymmetrical oscillations have irregular behavior. Interestingly, this test stimulated our colleague " $L$." to visit a cardiologist

Table 1. Pulse per minute and signal structure indicated for four persons using the blood pulse sensor

\begin{tabular}{l|l|l}
\hline Volunteer & Pulse/min & Signal structure \\
\hline J. & 66 & Regular oscillations \\
\hline V. & 71 & Regular oscillations \\
\hline Vl. & 62 & Regular nonsymmetrical oscillations \\
\hline L. & 94 & Irregular nonsymmetrical oscillations \\
\hline
\end{tabular}




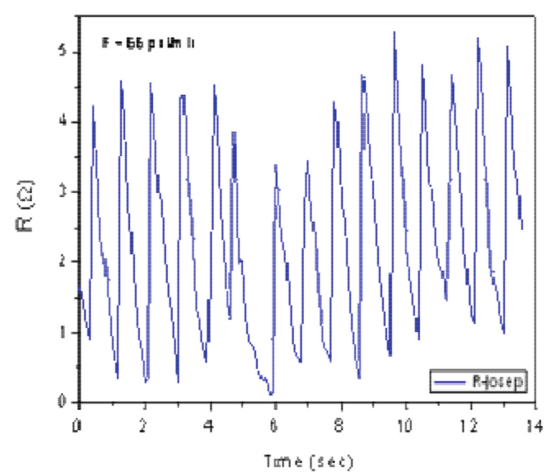

a)

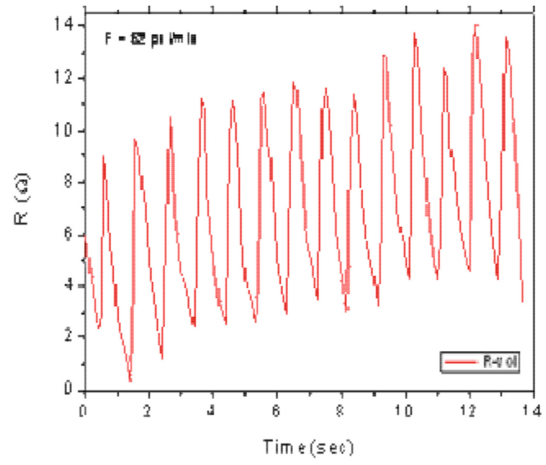

c)

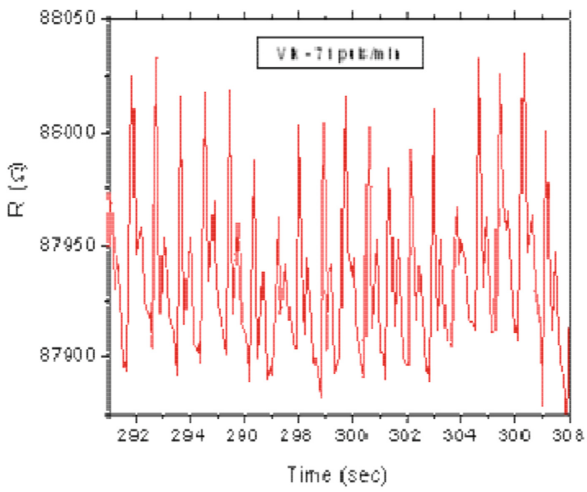

b)

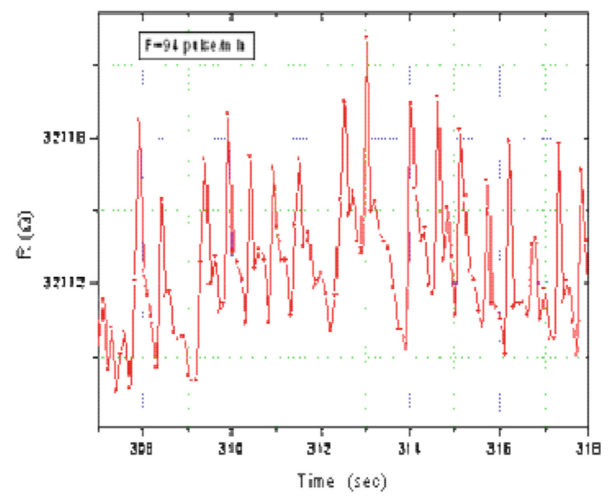

d)

Fig. 4. Resistance response to the blood-pulse movement measured for 4 different persons: (a) volunteer "J.", men, 27 years old, (b) volunteer "V.", men, 27 years old, (c) volunteer "Vl.", men, 64 years old, and (d) volunteer " $L$." men, 35 year old

who diagnosed that " $L$." has ciliary arrhythmia. Therefore, our sensor assist the medical personnel in controlling blood pulse and revealing cardiologic problems by a simple procedure.

\subsubsection{Intraocular Pressure Sensing Contact Lens}

The contact lens capable of Intraocular Pressure (IOP) monitoring in noninvasive way is presented in Fig. 2c. Among numerous attempts to continuously monitor the IOP in a non-invasive way [23, 24] only Leonardi et al. have developed a marketable device [25]. The key element of the device is a soft contact lens with an embedded microfabricated strain gauge (platinum-titanium foil) allowing the measurement of changes in corneal curvature correlated to variations in IOP. We have developed the IOP sensing devices based on rigid and hybrid contact lenses equipped with flexible sensing membranes fabricated from polycarbonate/(BEDT-TTF) ${ }_{2} \mathrm{I}_{3}$ [26].

The developed devices (Fig. 5) use a different measure approach and, moreover, the gauge factor of the piezoresistor: polycarbonate/(BEDT-TTF $)_{2} \mathrm{I}_{3}$ is five times larger 


\section{Cup-like protection}

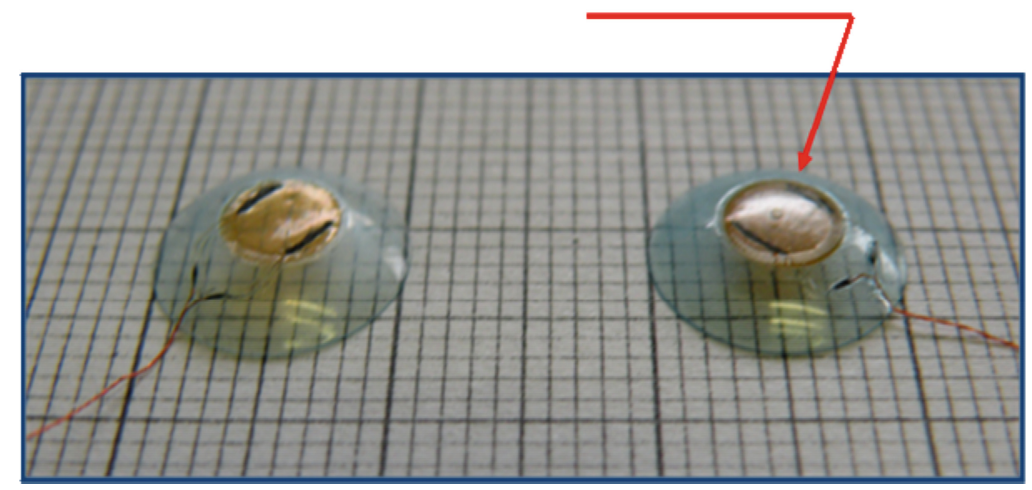

Fig. 5. Photo image of contact IOP sensing lenses without (left) and with (right) the top cap protection of the strain BL film based-sensor showing the wires for measuring the resistance changes

than the platinum gauge factor. All pressure tests are prepared using hand-made set-up. The contact lens based sensor was pasted over ring-like glass holder that can be connected by the tube to the Low-Pressure Controller CPC2000 ("Mensor"). The tests showed that our BL sensor is able to detect deformations in the corneal curvature caused by pressure changes as small as 1-2 mbar. The electrical response of the developed lenses to pressure changes reveals a proper sensitivity to perform continuous monitoring of IOP (Fig. 6).

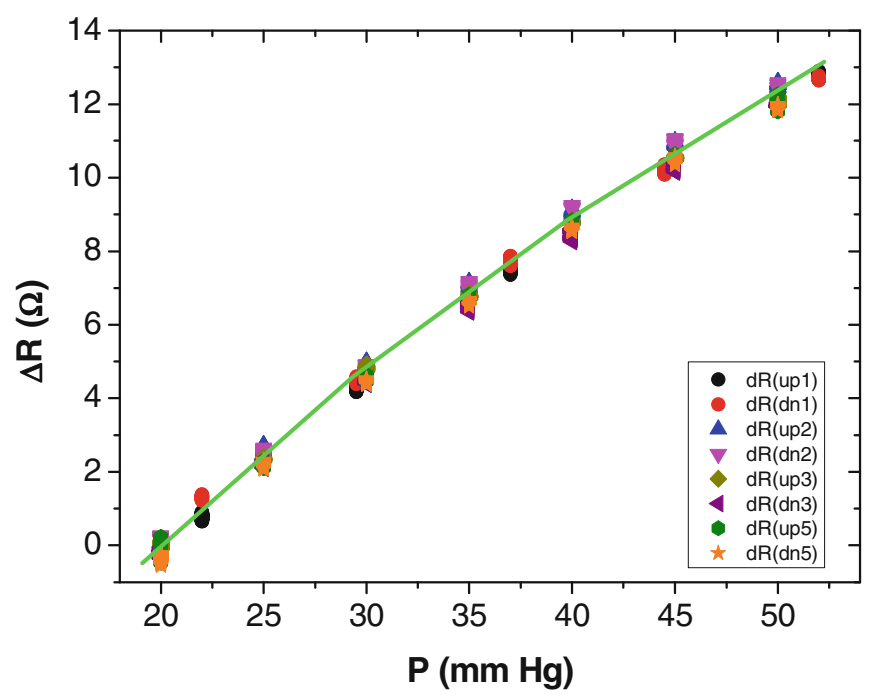

Fig. 6. Typical electrical response of the developed sensing lenses to IOP changes; data collected for four up-down sweeps 


\subsection{Temperature Sensing Device}

Temperature is one of the crucial parameters to be measured in numerous monitoring applications. Healthcare, environment control, biomedical applications call for low cost, lightweight thermistors which might accurately measure temperature changes. This challenge prompted us to apply the BEDT-TTF-based conductors to engineering low cost, lightweight thermistor whose resistance will be able to respond to small temperature changes. For this purpose we propose to use the recently developed BL thermistor: polycarbonate/ $\alpha^{\prime}-(\mathrm{BEDT}-\mathrm{TTF})_{2} \mathrm{I}_{\mathrm{x}} \mathrm{Br}_{3-\mathrm{x}}$, where $\alpha^{\prime}$-(BEDT-TTF) $)_{2} \mathrm{I}_{\mathrm{x}} \mathrm{Br}_{3-\mathrm{x}}$ is organic molecular semiconductor whose temperature coefficient of resistance (TCR) was found as $-1.4 \% /$ degree. A sample of the conductive BL film: polycarbonate/ $\alpha$ '-(BEDTTTF) ${ }_{2} \mathrm{I}_{\mathrm{x}} \mathrm{Br}_{3-\mathrm{x}}$ with a sensing area around $2 \times 3 \mathrm{~mm}^{2}$ and thickness $10-30 \mu \mathrm{m}$ (including temperature sensing layer: $(0.5$ to $1 \mu \mathrm{m})$ is located over a ridged support. Four Pt wires are connected to the sensing element using graphite paste as it shown in Fig. 7.

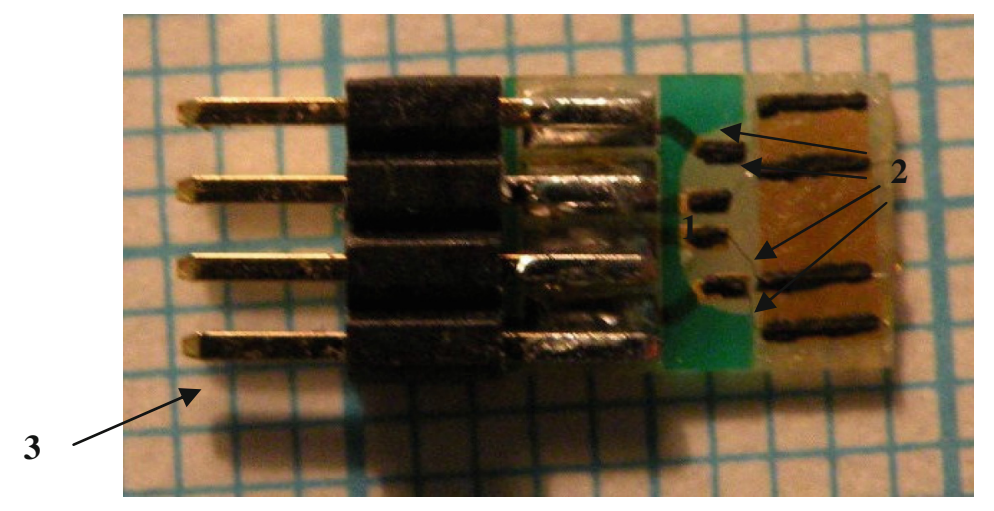

Fig. 7. Photo image of temperature sensing device: 1 - BL thermister: polycarbonate/ $\alpha$ '$(\mathrm{BEDT}-\mathrm{TTF})_{2} \mathrm{I}_{\mathrm{x}} \mathrm{Br}_{3-\mathrm{x}}, 2$ - graphite contacts, 3 - connector

We note here that sensing area can be adjusted according to the sensing application requirements. Some important data on sensor testing are summarized in the following paragraph:

- range of ice point resistance: $\mathrm{R}=10-20 \mathrm{k} \Omega$ for a sensing area $2 \times 3 \mathrm{~mm}^{2}$;

- temperature range: $-50{ }^{\circ} \mathrm{C}$ to $80{ }^{\circ} \mathrm{C}$; thermistor shows a weak semiconductor-like behavior;

- resolution is about: $0.001{ }^{\circ} \mathrm{C}$;

- fundamental temperature interval: $-10{ }^{\circ} \mathrm{C}$ to $40{ }^{\circ} \mathrm{C}$;

- self heating: $<0.1{ }^{\circ} \mathrm{C} / \mathrm{mW}$;

- thermal response: $<0.1 \mathrm{~s}$.

- the power needed for the measurement: $1-5 \mu \mathrm{W}$ (current $10 \mu \mathrm{A}$ ).

Taking into account that temperature of water in ocean and others natural water reservoirs is very important characteristic, which is the major factor governing the 

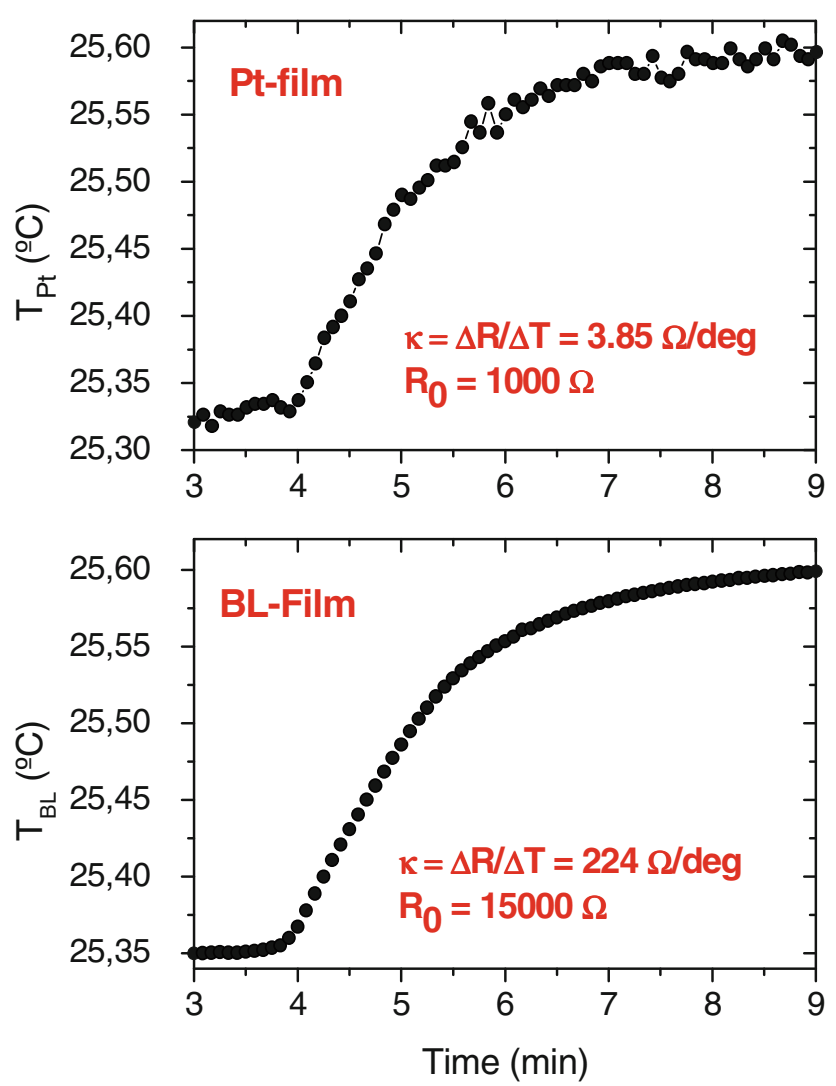

Fig. 8. Temperature control using (top) Pt Film Detector: Pt1000 (RS order code: 362-9907, Dimensions: $2.0 \times 10 \mathrm{~mm}^{2}$ ) and (bottom) developed detector with BL thermister: polycarbonate/ $\alpha$ '-(BEDT-TTF) ${ }_{2} \mathrm{I}_{\mathrm{x}} \mathrm{Br}_{3-\mathrm{x}}$

vertical movement of ocean waters [27], we studied the resistance temperature dependence of the fabricated device in water (Fig. 8, bottom).

For the sake of verification of obtained results, we experimentally evaluate them with respect to commonly used Pt-based thermometer (Fig. 8, top). According to this comparative study, the developed temperature sensing device far superior in sensitivity ( $k$ ) the commercial Pt-based thermometer: for the developed sensing device the value of $k$ was found as $224 \Omega /$ degree while for Pt thermometer it is $3.85 \Omega /$ degree.

\section{Exploring Organic Film Sensors and Wireless Sensor Network Paradigm}

As we noticed earlier, there is a growing interest in film based electronic components, e.g. sensors and storage [28], which can be deposited right on the surface of the embedded systems. The reason for this interest is grounded on newly available 
materials, which can ensure better performance, on full customization of printed/ deposited devices and, in most cases, on a simple fabrication process. However, there is still a gap between the printed devices and their integration with embedded electronics and, in particular, with WSNs. In fact the WSN paradigm is considered as a pillar technology in the forthcoming era of the Internet of Things (IoT) [29, 30]. The IoT paradigm aims at intelligent connecting devices, e.g. sensors and actuators [32], and services in a global network. This approach will help to resolve a number of problems including a remote medical assistance one which appears to be an important challenge in a growing urban areas. The IoT devices equipped with the wearable sensors presented in this work will enable the 'telemedicine' option. This approach will help the medical personnel to perform the remote assistance and consultation for a number of patients.

In this section we demonstrate how to interface a film sensor with a custom made wireless sensor node and conduct temperature measurement in real settings. We note that the sensor node is developed for the 'proof of concept' purpose only.

\subsection{Interfacing of Sensing Device and Sensor Node}

The architecture of the sensor node, shown in Fig. 9, includes four main blocks: processing, sensing, communication, and power management. The processing unit, based on an ATXmega128 Microprocessor Control Unit (MCU) with a precise 24-bit Analog-to-Digital Converter (ADC), manages the operation of the sensors and of the ETRX3 wireless modem capable of transmitting and receiving data. ETRX3 has a number of self-configuration options, e.g. network configuration, adjustment of transmit power, which ensure that the WSN can be deployed and debugged in short time. The sensing unit includes the temperature and humidity film sensors. The sensors advantages over off-the-shelf components are quick response time and high sensitivity. Power management provides the node with $3 \mathrm{~V}$ of supply voltage. As battery, a $3.6 \mathrm{~V}$ AA-size Li-ion cell can be used or two alkaline/NiMH $1.5 \mathrm{~V}$ each AA-size cells can be applied and wired in series (the first option is preferable due to higher energy density). In this work we use $3.6 \mathrm{~V} \mathrm{Li}$-ion AA-type battery with $3200 \mathrm{mAh}$ capacity.

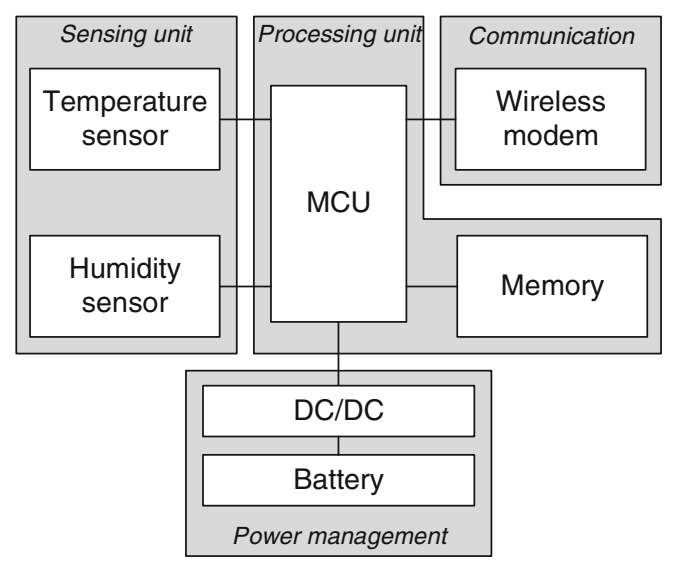

Fig. 9. Sensor node architecture 


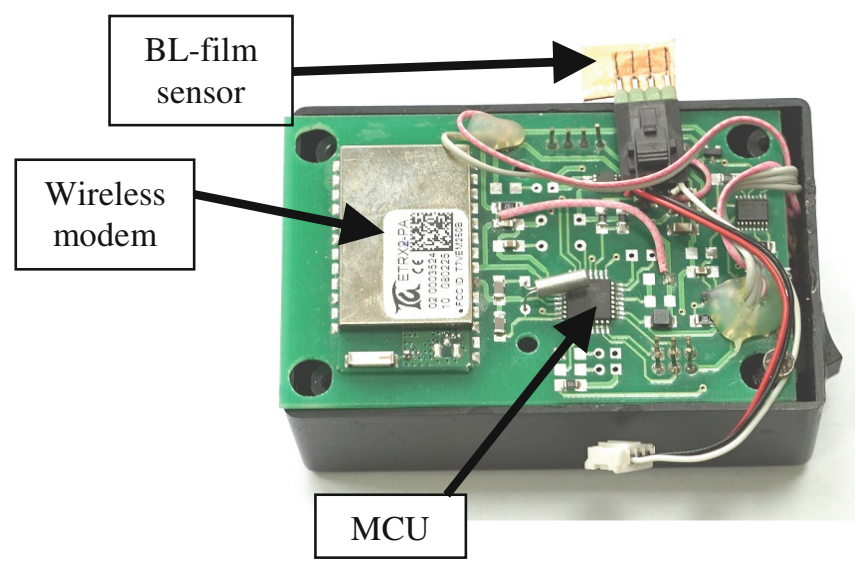

Fig. 10. Prototype of the wireless sensor node with the BL-film as a sensing element.

The prototype of the wireless sensor node with BL film is shown on Fig. 10. The node prototype is featured by a compact design $(8 \times 4.5 \mathrm{~cm})$, the wireless communication capability and BL-film sensor.

Except for the sensors, which were fabricated in our laboratory the electronic components used in this design are off-the-shelf. These components were chosen with a special focus on their low power consumption which is highly important requirement for WSN devices [31]. The novel aspect of the sensor design is the use of "self-metallization" technology that allows the temperature and humidity sensors to be deposited in the empty spaces of the board. In the prototype, we have used an extra board for our testing convenience.

\subsection{Experimental Results}

In this section, we experimentally evaluate the film sensor performance in terms of response time and compare the result with a similar off-the-shelf component and present the sensor behavior in a real scenario.

The typical resistance response of the polycarbonate/(BEDT-TTF $)_{2} \mathrm{Br}_{\mathrm{x}}\left(\mathrm{H}_{2} \mathrm{O}\right)_{\mathrm{n}}$ bilayer film to relative humidity $(\mathrm{RH})$ changes is shown in Fig. 11. It shows how the internal resistance of the sensor changes as the temperature changes. The results indicate that the electrical response of the polycarbonate/ $\alpha^{\prime}-(\mathrm{BEDT}-\mathrm{TTF})_{2} \mathrm{I}_{\mathrm{x}} \mathrm{Br}_{3-\mathrm{x}} \mathrm{BL}$ film to temperature changes is reversible and a well reproducible signal. Moreover, this response is as fast as the response of thermometer Pt110.

To calibrate the sensor we apply the supply voltage to the leftmost and rightmost contacts of the sample (see Fig. 7). It generates the current flowing across. The measurement of this current is performed by connecting a precise resistor of $1 \Omega$ in series with the film and measuring its voltage by ADC. Next we measure the voltage between two central contacts of the sensor using ADC and calculate the resistance using the Ohm's law and taking into consideration the resistance of the contacts. Since the calibration is conducted in a climatic chamber we associate the temperature with 


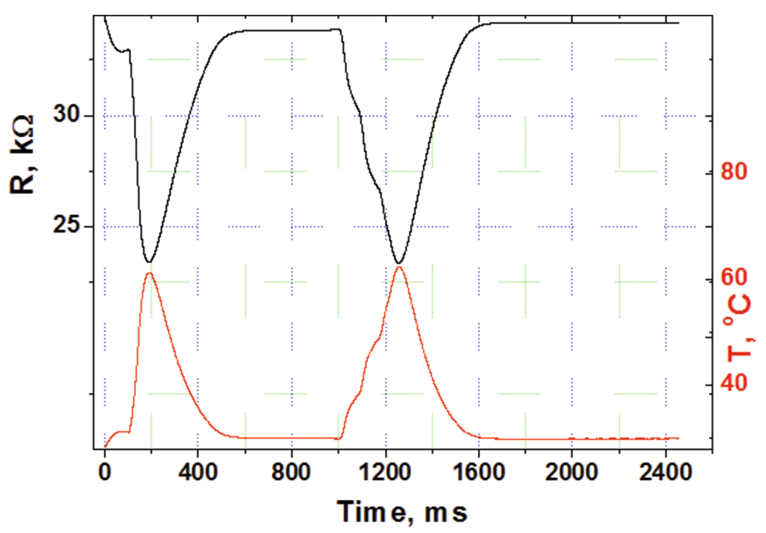

Fig. 11. Sensor performance: resistance of the polycarbonate/ $\alpha$ '-(BEDT-TTF) ${ }_{2} \mathrm{I}_{\mathrm{x}} \mathrm{Br}_{3-\mathrm{x}} \mathrm{BL}$ film (black) and temperature (measured by reference thermometer Pt110) changes (red) over time (Color figure online)

resistance and program the table with these values in the memory of MCU. The film sensor resistance is a non linear function of temperature and humidity that is why we perform ten measurements of resistance at a know temperature, calculate the average value and, finally, associate this value with the temperature value.

Figure 12 demonstrates how the film resistance and ambient temperature change in an office environment during working hours. In this experiment we have used the wireless sensor node with the BL-film sensor for the evaluation of the ambient temperature. The sensor node conducts the measurements once per five minutes and sends the measured data to the coordinator once an hour for the sake of energy savings. The results demonstrate that even the temperature is kept at a particular temperature level in the building by a Heating, Ventilation and Air Conditioning (HVAC) system, the temperature in the office varies. We infer that the temperature grows in the beginning of working day (by 9:00) since the office workers arrive to their office place and gradually decreases by the lunch time (around 13:00). Then the temperature increase while people come back to the office and slows down by the end of the working day.

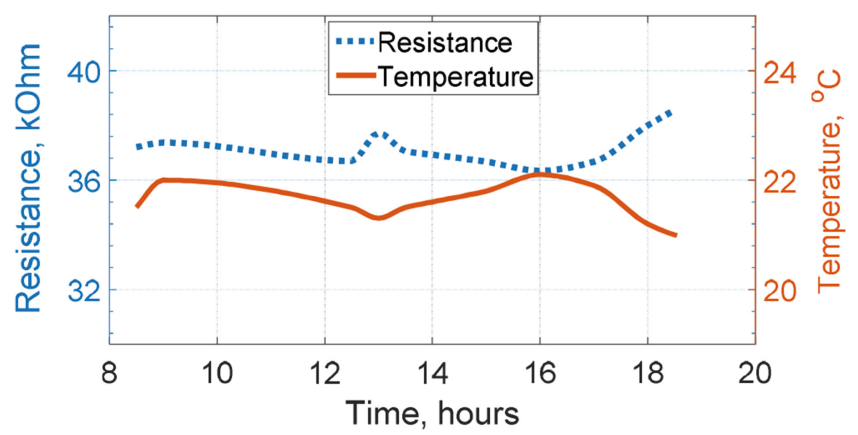

Fig. 12. Experiment showing how the film resistance and temperature change in an office environment during the working hours. 


\section{Conclusions}

In this work we have demonstrated the sensing devices for biomedical applications equipped with flexible either BL piezoresitors or BL thermistors. The carried out proof-of-concept experiments demonstrate that developed sensing devices far superior in sensitivity their commercial off-the-shelf analogues and they are able to detect even negligible change in pressure and temperature changes by measuring the sensors' response signals.

Also we have demonstrated how the proposed temperature sensor can be interfaced with a wireless sensor node. The experimental results demonstrate that the obtained values of the temperature coefficient of resistance for the organic films is four times higher than the value of the platinum which results in better film performance. Apart from this, the films are characterised by cheap manufacturing process and film customization in terms of its shape and resistance which helps to use the unoccupied surfaces of the sensor node.

Our future work includes the investigation of sensor measurements stability and application of sensors in wearable scenarios.

Acknowledgments. The authors acknowledge the financial support from Instituto de Salud Carlos III, through "Acciones CIBER.” The Networking Research Center on Bioengineering, Biomaterials and Nanomedicine (CIBER-BBN), an initiative funded by theVINational R\&D\&I Plan 2008-2011, Iniciativa Ingenio 2010, Consolider Program, CIBER Actions and financed by the Instituto de Salud Carlos IIIwith assistance from the European Regional Development Fund. The authors also appreciate the financial support through the projects: BE-WELL (CTQ201340480-R) granted by DGI (Spain), and GenCat (2014-SGR-17) financed by DGR (Catalunya), the European Commission's Seventh Framework Programme for Research under contracts FP7-OCEAN-2013-614155, the Ministry of Education and Science of Russian Federation Grant RFMEFI57714X0133.

\section{References}

1. Lorussi, F., Rocchia, W., Scilingo, E.P., Tognetti, A., De Rossi, D.: Wearable, redundant fabric-based sensor arrays for reconstruction of body segment posture. IEEE Sens. J. 4, 807818 (2004)

2. Stoppa, M., Chiolerio, A.: Sensors wearable electronics and smart textiles: a critical review. Sensors 14, 11957-11992 (2014)

3. Lumelsky, V.J., Shur, M.S., Wagner, S.: Sensitive skin. IEEE Sens. J. 1, 41-51 (2001)

4. Dyo, V., Ellwood, S.A., Macdonald, D.W., Markham, A., Trigoni, N., Wohlers, R., Mascolo, C., Pásztor, B., Scellato, S., Yousef, K.: WILDSENSING: design and deployment of a sustainable sensor network for wildlife monitoring. ACM Trans. Sens. Netw. 8(4), 1-33 (2012). Article 29

5. Somov, A., Spirjakin, D., Ivanov, M., Khromushin, I., Passerone, R., Baranov, A., Savkin, A.: Combustible gases and early fire detection: an autonomous system for wireless sensor networks. In: Proceeding of e-Energy, pp. 85-93 (2010)

6. Doolin, D.M., Sitar, N.: Wireless sensors for wildfire monitoring. In: Proceedings of Sensors and Smart Structures Technologies for Civil, Mechanical, and Aerospace Systems, San Diego, CA, USA, vol. 5765 (2005) 
7. Segura-Garcia, J., Felici-Castell, S., Perez-Solano, J.J., Cobos, M., Navarro, J.M.: Low-cost alternatives for urban noise nuisance monitoring using wireless sensor networks. IEEE Sens. J. 15, 836-844 (2015)

8. Dutta, T.: Medical data compression and transmission in wireless ad hoc networks. J. IEEE Sens. 15, 778-786 (2015)

9. Mittal, K.L.: Preface in Metallized Plastics 7: Fundamental and Applied Aspects, p. vii. VSP BV, Utrecht (2001). Mittal, K.L. (ed.)

10. Huang, C.-C., Kao, Z.-K., Liao, Y.-C.: Flexible miniaturized nickel oxide thermistor arrays via inkjet printing technology. ACS Appl. Mater. Interfaces 5(24), 12954-12959 (2013)

11. Waltman, R.J., Bargon, J.: Electrically conducting polymers: a review of the electropolymerization reaction, of the effects of chemical structure on polymer film properties, and of applications towards technology. Can. J. Chem. 64, 76-95 (1986)

12. Mahadeva, S.K., Yun, S., Jaehwan, K.: Flexible humidity and temperature sensor based on cellulose-polypyrrole nanocomposite. Sens. Actuators A: Phys. 165(2), 194-199 (2011)

13. Cardoso, M.J.R., Lima, M., Lenz, D.M.: Polyaniline synthesized with functionalized sulfonic acids for blends manufacture. Mater. Res. 10(4), 425-429 (2007)

14. Shibaeva, R.P., Yagubskii, E.B.: Molecular conductors and superconductors based on trihalides of BEDT-TTF and some of its analogues. Chem. Rev. 104, 5347-5378 (2004)

15. Saito, G.: Chap. 10. In: Jones, W. (ed.) Organic Molecular Solids. CRC, Boca Raton (1997)

16. Laukhina, E., Rovira, C., Ulanski, J.: Organic metals as active components in surface conducting semitransparent films. Synth. Met. 21, 1407-1408 (2001)

17. Laukhina, E., Ulanski, J., Khomenko, A., Pesotskii, S., Tkacheva, V., Atovmyan, L., Yagubskii, E., Rovira, C., Veciana, J., Vidal-Gancedo, J., Laukhin, V.: Systematic study of the $(\mathrm{ET})_{2} \mathrm{I}_{3}$ reticulate doped polycarbonate film: structure, ESR, transport properties and superconductivity. J. Phys. I Fr. 7, 1665-1675 (1997)

18. Laukhina, E., Pfattner, R., Ferreras, L.R., Galli, S., Mas-Torrent, M., Masciocchi, N., Laukhin, V., Rovira, C., Veciana, J.: Ultrasensitive piezoresistive all-organic flexible thin films. Adv. Mater. 22, 977-981 (2010)

19. Lebedev, V., Laukhina, E., Laukhin, V., Rovira, C., Veciana, J.: Tuning the electronic properties of piezoresistive bilayer films based on alpha-(BEDT-TTF) $)_{2} \mathrm{I}_{3}$. Eur. J. Inorg. Chem. 2014, 3927-3932 (2014)

20. Laukhina, E., Tkacheva, V., Khasanov, S., Zorina, L., Gomez-Segura, J., Perezdel Pino, A., Veciana, J., Laukhin, V., Rovira, C.: Linked crystallites in the conducting topmost layer of polymer bilayer films controlled by temperature: from micro- to nanocrystallites. ChemPhysChem 7, 920-923 (2006)

21. PT-100 Series Platinum RTDs. http://www.lakeshore.com/Documents/LSTC_Platinum_l. pdf

22. Lebedev, V., Laukhina, E., Rovira, C., Laukhin, V., Veciana, J.: All-organic humidity sensing films with electrical detection principle suitable to biomedical applications. Procedia Eng. 47, 603-606 (2012)

23. Maurice, D.M.: A recording tonometer. Brit. J. Ophthal 42, 321-335 (1958)

24. Svedbergh, B., Bäcklund, Y., Hök, B., Rosengren, L.: The IOP-IOL: a probe into the eye. Acta Ophthalmol. 70(2), 266-268 (1992)

25. Leonardi, M., Pitchon, E.M., Bertsch, A., Renaud, P., Mermoud, A.: Wireless contact lens sensor for intraocular pressure monitoring: assessment on enucleated pig eyes. Acta Ophthalmol. 87, 433-437 (2009)

26. Laukhin, V., Lebedev, V., Laukhina, E., Martin, R., Pastor, J.C., Villa, R., Aguilo, J., Rovira, C., Veciana, J.: Hybrid contact lens capable of intraocular pressure monitoring in noninvasive way. In: Solid-State Sensors, Actuators and Microsystems (Transducers and Eurosensors XXVII), pp. 1871-1874 (2013) 
27. http://ocean.stanford.edu/courses/bomc/chem/lecture_03.pdf

28. Somov, A., Ho, C.C., Passerone, R., Evans, J.W., Wright, P.K.: Towards extending sensor node lifetime with printed supercapacitors. In: Picco, G.P., Heinzelman, W. (eds.) EWSN 2012. LNCS, vol. 7158, pp. 212-227. Springer, Heidelberg (2012)

29. Miorandi, D., Sicari, S., De Pellegrini, F., Chlamtac, I.: Internet of Things: vision, applications and research challenges. Ad Hoc Netw. 10, 1497-1516 (2012)

30. Kelaidonis, D., Somov, A., Foteinos, V., Poulios, G., Stavroulaki, V., Vlacheas, P., Demestichas, P., Baranov, A., Biswas, A.R., Giaffreda, R.: Virtualization and cognitive management of real world objects in the Internet of Things. In: IEEE International Conference on Green Computing and Communications (GreenCom), pp. 187-194. IEEE Press (2012)

31. Somov, A., Minakov, I., Simalatsar, A., Fontana, G., Passerone, R.: A methodology for power consumption evaluation of wireless sensor networks. In: IEEE Conference on Emerging Technologies and Factory Automation (ETFA 2009), pp. 1-8. IEEE Press (2009)

32. Somov, A., Baranov, A., Spirjakin, D.: A wireless sensor-actuator system for hazardous gases detection and control. J. Sens. Actuators A: Phys. 210, 157-164 (2014) 